\title{
THE ROLE OF UTERINE INFECTION IN PREGNANCY OUTCOME: A
} CASE REPORT

\section{Tatyana T. Bodurska, Emiliana I. Konova ${ }^{1}$, Svetlana Pachkova, Angel D. Yordanov ${ }^{2}$}

\author{
Medical Center, Clinical Institute for \\ Reproductive Medicine - Pleven \\ ${ }^{1}$ Center for reproductive health, \\ Medical University - Pleven \\ ${ }^{2}$ Department of Gynecologic Oncology, \\ Medical University - Pleven
}

\section{Summary}

It was believed in the past that only the lower genital tract is inhabited by microorganisms, and the cervix was considered as a perfect barrier against the ascension of microbes. Nowadays, many studies using new sequencing technologies examining microbiomes with low biomass show that the microorganisms are gradually changing from the lower to the upper genital tract with decreasing bacterial abundance and increasing bacterial diversity. It is well documented that the endometrium is a non-sterile area, and in healthy women, the Lactobacillus spp. is the dominant flora. Lactobacillus as a dominant endometrial microbiome, has a crucial role in implantation, early pregnancy development. It is also essential for a good pregnancy outcome. Non-Lactobacillusdominant microbiome is associated with poor reproductive outcomes (recurrent implantation failure, recurrent miscarriages, outgoing pregnancy). This association is evidence of its significance for reproductive health.

We present a case of unsuccessful first pregnancy with concurrent bacterial endometrial infection. A definitive antibiotic treatment resulted in a successful full-term second pregnancy. We present the influence of pathogenic microorganisms on uterine function and pregnancy development, current possibilities for diagnosis and treatment.

Keywords: endometrial microbiome, Lactobacillus, endometritis, plasmatic cells, antibiotics

\section{Introduction}

Since recently, the uterus has been assumed as a nonsterile organ [1]. For a long time, it was thought that the microorganisms in the uterus were present only in case of infection or contamination [2]. Currently, many studies using new sequencing technologies to examine microbiomes with low biomass show that the microorganisms are gradually ascending from the lower to the upper genital tract with decreasing bacterial abundance and increasing bacterial diversity [3-5]. In healthy women, the Lactobacillus spp. is the dominant flora in the endometrium [6]. It plays a crucial role in implantation, early pregnancy development and is essential for normal pregnancy outcomes [7]. Recent studies using next-generation sequencing (NGS) have

\author{
Received: July 29, 2021 \\ Revision received: August 20, 2021 \\ Accepted: November 25, 2021 \\ Corresponding Author: \\ Department of Gynecologic Oncology, \\ Medical University - Pleven \\ Pleven, 5800 \\ e-mail: angel.jordanov@gmail.com
}


detected unique uterine microbial composition [8]. Some studies show a correlation between endometrial microbiome and reproductive outcome in assisted reproduction [7,9-16], major gynecological diseases such as chronic endometritis [14,17], endometriosis [18-19], dysfunctional endometrial bleeding [20], endometrial polyps [17], endometrial hyperplasia, and cancer [3]. The dominant microorganisms in the uterine cavity in healthy women are Lactobacilli [6]. They play a crucial role in implantation, early pregnancy development, and pregnancy outcome. The presence of commensal bacteria on mucosa lining in homeostasis with host-organism develops resistance against pathogenic colonization [21].

Regarding the uterus, a cross-regulation between the uterine microbiome and local immune system is suggested. This symbiosis is essential for maintaining a balanced inflammatory environment which is potentially favorable for embryo implantation. On the other hand, regulation of the immune system by the endometrial microbiome induces tolerance to non-sterile semen and paternal antigens on the embryo.

\section{Clinical Case}

We present a case report of unsuccessful firsttrimester pregnancy followed by successful second pregnancy after investigation and treatment. A 29-year-old primigravida nullipara was diagnosed with missed abortion at 8-9 weeks of gestation. After dilatation and curettage, karyotyping and histological examination of abortive material were done. Karyotype excluded the most common trisomies - trisomy 21,13 , and 18. On histological examination, purulent exudate was found. Post-curettage antibiotic prophylaxis included intravenous application of cephalosporins. Other medical tests showed normal thyroid function, normal ovarian reserve, normal vaginal and cervical microbiome, negative antiphospholipid screen, normal semen analysis. The patient is a homozygote carrier on mutant allele in methylenetetrahydrofolate reductase gene.

Three months later, an endometrial biopsy was performed. A significant amount of Staphylococcus spp. (39-53\%) were found on real-PCR examination. Also, the amount of
Mobiluncus spp. and Corynebacterium spp. was substantial $(44-60 \%)$. Atopobium vaginae was detected in a small amount (1.1-1.4\%). Lactobacilli were not detected. Quantitative analysis of plasmatic cells in endometrial biopsy revealed an increased amount consistent with chronic endometritis. Peroral treatment with moxifloxacin for 10 days, peroral and vaginal lactobacilli were prescribed. One month after treatment control endometrial biopsy was performed. Staphylococcus spp. was not detected. Plasmatic cell level was normal. Atopobium vaginae level was increased (3547\%) compared with the previous examination. Peroral treatment with clindamycin and peroral and vaginal lactobacilli was prescribed. No other control endometrial biopsy was performed. One month later, peroral drugs for induction of ovulation were prescribed, and on the second cycle, the patient got pregnant. Uneventful pregnancy, full-term baby $3650 \mathrm{~g}, 52 \mathrm{~cm}$ was delivered vaginally. The puerperal period was normal.

\section{Discussion}

The dominant flora in the female genital tract is composed of Lactobacilli. Lactobacilli are prevalent not only in the lower genital tract but also in the upper genital tract. In a study by Mitchell et al., bacterial cultures from endometrial samples were detected in $100 \%$, with lactobacilli being dominant, followed by Gardnerella, Prevotella, Atopobium, and Sneathia, which were also found in vaginal samples [6]. Compared with humans, the genital tract of other mammalians is not inhabited by lactobacilli [22].

Vaginal lactobacilli produce lactic acid and short-chain fatty acids, causing lower $\mathrm{pH}$ levels. However, this is not the case in the uterine cavity. The $\mathrm{pH}$ in the endometrium is $6.6-8.51$. These data suggest other biochemical processes in the endometrium, where the embryo adheres and develops. Non-Lactobacillus dominant microbiome triggers an inflammatory process in the endometrium, thus affecting embryo implantation because inflammatory mediators are tightly regulated in the blastocyst adhesion to the endometrial wall [23]. Uterine infection is a known factor in infertility. The pathogenic 
environment leads to inflammation and immune activation of the endometrium, preventing embryo implantation and the beginning of a successful pregnancy. Verstraelen et al. examined the endometrial microbiome in patients with recurrent implantation failure and recurrent miscarriages or both. They found dysbiosis with a shift in the ratio of lactobacilli-dominant environment towards missing or reduced lactobacilli environment [16]. T-regulatory cells are essential for embryo implantation and early placental development [24-25]. Moore et al. established a higher percentage of births in cases of lactobacilli present at the tip of the embryo transfer catheter, which supports the essential role of the intrauterine commensal bacteria in maintaining immunological homeostasis, stimulating mucosal immunity, and preventing excessive inflammation [26]. The high stability of the vaginal microbiota during pregnancy [27] protects against the ascension of pathological microorganisms from the vagina to the maternal-fetal interface, preventing pregnancy damage [28]. More data on the importance of the microbiome are now available. Moreno et al. found that Lactobacillus was negatively correlated to pathogenic microorganisms and positively correlated to commensal bacteria, which may play a role in stability in the ecosystem [29]. They concluded that the pathogenic profile in patients with reproductive failure consists of Atopobium, Bifidobacterium, Chryseobacterium, Gardnerella, Haemophilus, Klebsiella, Neisseria, Staphylococcus, and Streptococcus. Staphylococcus was significantly increased in endometrial fluid in patients with clinical miscarriage. Staphylococcus is one of the main pathogenic bacteria reported to cause chronic endometritis.

The patient we present had chronic endometritis caused by Staphylococcus spp. and no lactobacilli in the endometrium. After definitive antibiotic treatment against a known bacterial agent and control endometrial biopsy to verify the treatment result, the patient got pregnant, and the pregnancy was uneventful. In this case, we can speculate that the definitive antibiotic treatment has a beneficial effect on the second pregnancy. However, it is well known that a normal pregnancy is a complex process affected by many internal and external factors.
We want to demonstrate the importance of the endometrial environment on early pregnancy development. As mentioned above, a lot of data is accumulated nowadays showing the effect of plasmatic cells, pathogenic microorganisms, including bacteria, viruses, fungi, and commensal like Lactobacilli on implantation, early pregnancy development, and late pregnancy complications.

\section{Conclusion}

Pregnancy is a complex process. A lot of factors may affect embryo adhesion and implantation, and early pregnancy development. More data on the effect of the uterine microbiome are needed, although current data show its implication on pregnancy outcomes. These data will be of great value for women with infertility and reproductive failure and women with other gynecological diseases and for maintaining gynecological health.

\section{References}

1. Tissier H. Recherches sur la Flore Intestinale des Nourrissons (e'tat Normal et Pathologique). G Carre and C Naud, Paris, 1900.

2. Ansbacher R, Boyson WA, Morris JA. Sterility of the uterine cavity. Am J Obstet Gynecol. 1967;99(3):394-6.

3. Walther-António MRS, Chen J, Multinu F, Hokenstad A, Distad TJ, Cheek EH, et al. Potential contribution of the uterine microbiome in the development of endometrial cancer. Genome Med. 2016;8(1):1-15.

4. Chen C, Song X, Wei W, Zhong H, Dai J, Lan Z, et al. The microbiota continuum along the female reproductive tract and its relation to uterinerelated diseases. Nat Commun. 2017;8(1).

5. Peric A, Weiss J, Vulliemoz N, Baud D, Stojanov M. Bacterial Colonization of the Female Upper Genital Tract. Int J Mol Sci. 2019;20(14):3405.

6. Mitchell CM, Haick A, Nkwopara E, Garcia R, Rendi M, Agnew K, et al. Colonization of the upper genital tract by vaginal bacterial species in nonpregnant women. Am J Obstet Gynecol. 2015;212(5):611.e1-611.e9.

7. Moreno I, Codoñer FM, Vilella F, Valbuena D, Martinez-Blanch JF, Jimenez-Almazán J, et al. Evidence that the endometrial microbiota has an effect on implantation success or failure. Am J Obstet Gynecol. 2016;215(6):684-703.

8. Giudice LC. Challenging dogma: the 
endometrium has a microbiome with functional consequences! Am J Obstet Gynecol. 2016;215(6):682-3.

9. Franasiak JM, Werner MD, Juneau CR, Tao X, Landis J, Zhan Y, et al. Endometrial microbiome at the time of embryo transfer: next-generation sequencing of the $16 \mathrm{~S}$ ribosomal subunit. J Assist Reprod Genet. 2016;33(1):129-36.

10. Hashimoto T, Kyono K. Does dysbiotic endometrium affect blastocyst implantation in IVF patients? J Assist Reprod Genet. 2019;36(12):2471-9.

11. Kitaya K, Nagai Y, Arai W, Sakuraba Y, Ishikawa T. Characterization of Microbiota in Endometrial Fluid and Vaginal Secretions in Infertile Women with Repeated Implantation Failure. Mediators Inflamm. 2019;2019:4893437.

12. Kyono K, Hashimoto T, Nagai $\mathrm{Y}$, Sakuraba Y. Analysis of endometrial microbiota by $16 \mathrm{~S}$ ribosomal RNA gene sequencing among infertile patients: a single-center pilot study. Reprod Med Biol. 2018;17(3):297-306.

13. Liu Y, Wong KK-W, Ko EY-L, Chen X, Huang J, Tsui SK-W, et al. Systematic Comparison of Bacterial Colonization of Endometrial Tissue and Fluid Samples in Recurrent Miscarriage Patients: Implications for Future Endometrial Microbiome Studies. Clin Chem. 2018;64(12):1743-52.

14. Moreno I, Cicinelli E, Garcia-Grau I, GonzalezMonfort M, Bau D, Vilella F, et al. The diagnosis of chronic endometritis in infertile asymptomatic women: a comparative study of histology, microbial cultures, hysteroscopy, and molecular microbiology. Am J Obstet Gynecol. 2018;218(6):602.e1-602.e16.

15. Wee BA, Thomas M, Sweeney EL, Frentiu FD, Samios M, Ravel J, et al. A retrospective pilot study to determine whether the reproductive tract microbiota differs between women with a history of infertility and fertile women. Aust New Zeal J Obstet Gynaecol. 2018;58(3):341-8.

16. Verstraelen H, Vilchez-Vargas R, Desimpel F, Jauregui R, Vankeirsbilck N, Weyers S, et al. Characterisation of the human uterine microbiome in non-pregnant women through deep sequencing of the V1-2 region of the $16 \mathrm{~S}$ rRNA gene. PeerJ. 2016;2016(1):1-23.

17. Fang R-L, Chen L-X, Shu W-S, Yao S-Z, Wang S-W, Chen Y-Q. Barcoded sequencing reveals diverse intrauterine microbiomes in patients suffering with endometrial polyps. Am J Transl Res. 2016;8(3):1581-92.

18. Hernandes C, Silveira P, Rodrigues Sereia AF, Christoff AP, Mendes H, Valter de Oliveira LF, et al. Microbiome Profile of Deep Endometriosis Patients: Comparison of Vaginal Fluid,
Endometrium and Lesion. Diagnostics (Basel, Switzerland). 2020;10(3):163.

19. Khan KN, Fujishita A, Masumoto H, Muto $\mathrm{H}$, Kitajima M, Masuzaki H, et al. Molecular detection of intrauterine microbial colonization in women with endometriosis. Eur J Obstet Gynecol Reprod Biol. 2016;199:69-75.

20. Pelzer ES, Willner D, Buttini M, Huygens F. A role for the endometrial microbiome in dysfunctional menstrual bleeding. Antonie Van Leeuwenhoek. 2018;111(6):933-43.

21. Schnupf P, Gaboriau-Routhiau V, CerfBensussan N. Modulation of the gut microbiota to improve innate resistance. Curr Opin Immunol. 2018;54:137-44.

22. Miller E, Beasley D, Dunn R, Archie E. Lactobacilli Dominance and Vaginal $\mathrm{pH}$ : Why is the Human Vaginal Microbiome Unique? Front Microbiol. 2016;7,1936.

23. Dominguez F, Gadea B, Mercader A, Esteban FJ, Pellicer A, Simón C. Embryologic outcome and secretome profile of implanted blastocysts obtained after coculture in human endometrial epithelial cells versus the sequential system. Fertil Steril. 2010;93(3):774-82.e1.

24. Shima T, Sasaki Y, Itoh M, Nakashima A, Ishii $\mathrm{N}$, Sugamura K, et al. Regulatory $\mathrm{T}$ cells are necessary for implantation and maintenance of early pregnancy but not late pregnancy in allogeneic mice. J Reprod Immunol. 2010;85(2):121-9.

25. Zhou J, Wang Z, Zhao X, Wang J, Sun H, Hu Y. An Increase of Treg Cells in the Peripheral Blood is Associated with a Better In Vitro Fertilization Treatment Outcome. Am J Reprod Immunol. 2012;68(2):100-6.

26. Moore DE, Soules MR, Klein NA, Fujimoto VY, Agnew KJ, Eschenbach DA. Bacteria in the transfer catheter tip influence the live-birth rate after in vitro fertilization. Fertil Steril. 2000;74(6):1118-24.

27. Walther-António MRS, Jeraldo P, Berg Miller ME, Yeoman CJ, Nelson KE, Wilson BA, et al. Pregnancy's Stronghold on the Vaginal Microbiome. PLoS One. 2014;9(6):e98514.

28. Romero R, Hassan SS, Gajer P, Tarca AL, Fadrosh DW, Nikita L, et al. Correction to: The composition and stability of the vaginal microbiota of normal pregnant women is different from that of non-pregnant women. Microbiome. 2014;2(1):1-19.

29. Moreno I, Garcia-Grau I, Perez-Villaroya D, Gonzalez-Monfort M, Bahçeci M, Barrionuevo MJ, et al. Endometrial microbiota composition is associated with reproductive outcome in infertile patients. medRxiv. 2021. DOI: https:// doi.org/10.1101/2021.02.05.21251207 